Research Paper

\title{
XPG Gene Polymorphisms Contribute to Colorectal Cancer Susceptibility: A Two-Stage Case-Control Study
}

\author{
Rui-Xi Hua ${ }^{1,3^{*}}$, Zhen-Jian Zhuo ${ }^{*}$, Jinhong Zhu ${ }^{5^{*}}$, Shao-Dan Zhang1, Wen-Qiong Xue ${ }^{1}$, Jiang-Bo Zhang1, \\ Hong-Mei $\mathrm{Xu}^{6}$, Xi-Zhao Li ${ }^{1}$, Pei-Fen Zhang ${ }^{1}$, Jing $\mathrm{He}^{1,2 凶}$, Wei-Hua Jia ${ }^{1 凶}$ \\ 1. Sun Yat-sen University Cancer Center, State Key Laboratory of Oncology in South China, Department of Experimental Research, Collaborative Innovation \\ Center for Cancer Medicine, Guangzhou 510060, Guangdong, China; \\ 2. Department of Pediatric Surgery, Guangzhou Institute of Pediatrics, Guangzhou Women and Children's Medical Center, Guangzhou Medical University, \\ Guangzhou 510623, Guangdong, China; \\ 3. Department of Oncology, The First Affiliated Hospital of Sun Yat-sen University, Guangzhou 510080, Guangdong, China: \\ 4. Guangdong Province Key Laboratory of Pharmacodynamic Constituents of TCM and New Drugs Research, College of Pharmacy, Jinan University, \\ Guangzhou 510632, Guangdong, China; \\ 5. Molecular Epidemiology Laboratory and Department of Laboratory Medicine, Harbin Medical University Cancer Hospital, Harbin 150040, Heilongjiang, \\ China; \\ 6. Reproductive Medical Center, Department of Obstetrics and Gynecology, Sun Yat-sen Memorial Hospital, Guangzhou 510120, Guangdong, China. \\ *These authors contributed equally to this work.
}

$\triangle$ Corresponding authors: Jing He, Sun Yat-sen University Cancer Center, State Key Laboratory of Oncology in South China, Department of Experimental Research, Collaborative Innovation Center for Cancer Medicine, 651 Dongfeng Road East, Guangzhou 510060, Guangdong, China. Tel./Fax: (+86-20) 87342410, Email: hejing27@mail.sysu.edu.cn, or Department of Pediatric Surgery, Guangzhou Institute of Pediatrics, Guangzhou Women and Children's Medical Center, Guangzhou Medical University, 9 Jinsui Road, Guangzhou 510623, Guangdong, China, Tel./Fax: (+86-020) 38076560, Email: hejing198374@gmail.com; or Wei-Hua Jia, Sun Yat-sen University Cancer Center State Key Laboratory of Oncology in South China, Department of Experimental Research, Collaborative Innovation Center for Cancer Medicine, 651 Dongfeng Road East, Guangzhou 510060, Guangdong, China, Tel.: (+86-20) 87342327; Fax: (+86-20) 87343392, Email: jiaweih@mail.sysu.edu.cn.

(0) Ivyspring International Publisher. Reproduction is permitted for personal, noncommercial use, provided that the article is in whole, unmodified, and properly cited. See http://ivyspring.com/terms for terms and conditions.

Received: 2016.03.21; Accepted: 2016.07.09; Published: 2016.08.06

\begin{abstract}
Previous studies have reported that xeroderma pigmentosum group $G(X P G)$ gene polymorphisms may modulate colorectal cancer (CRC) susceptibility. In this study, we performed a two-stage case-control study to comprehensively investigate the associations of five polymorphisms in the XPG gene with CRC risk in 1,901 cases and 1,976 controls from Southern China, including rs2094258 C>T, rs751402 C>T, rs2296147 T>C, rs1047768 T>C and rs873601 G>A. After combining data from two stages, we found that three of the studied polymorphisms (rs2094258 C>T, rs751402 C>T, and rs873601 G>A) were significantly associated with CRC susceptibility. After adjustment for age and gender, multivariate logistic regression analysis indicated that carriers of the rs2094258 T alleles had an increased CRC risk [CT vs. CC: adjusted odds ratio (OR)=1.17, $95 \%$ confidence interval $(\mathrm{Cl})=1.01-1.36 ; \mathrm{TT}$ vs. $\mathrm{CC}$ : adjusted $\mathrm{OR}=1.49,95 \% \mathrm{Cl}=1.18-1.89 ; \mathrm{TT}$ vs. CT/CC: adjusted $O R=1.38,95 \% \mathrm{Cl}=1.10-1.72]$. Likely, rs873601 A allele also conferred increased CRC susceptibility. In contrast, a protective association was identified between rs751402 C>T polymorphism and the risk of CRC. In summary, our results indicated that these three polymorphisms were found to associate with CRC susceptibility in a Southern Chinese population.
\end{abstract}

Key words: colorectal cancer; XPG; polymorphism; DNA repair; genetic susceptibility.

\section{Introduction}

Colorectal cancer $(\mathrm{CRC})$ is the third most commonly diagnosed cancer in men, while it second only to breast cancer in women. It was estimated that 1.4 million cases and 693,900 deaths might have occurred in 2012 [1]. Although CRC incidence rate has been thought to be less common in Asian than that in western countries [2], it has been greatly increasing in recent years, and imposed a substantial economic burden in China [3]. CRC is a multistep process, resulting from the interaction between alterations in 
an individual's genetic profile and exposures to environmental carcinogens [4]. Epidemiological studies have revealed that a number of factors may contribute to the etiology of CRC, including tobacco use, lack of exercise, overweight and obesity, high consumption of red meat, and alcohol intake. The fact that only a subset of individuals exposed to risk factors actually develop CRC in their lifetime suggests that the role of genetic factors is also indispensible in the development of CRC [5-13].

Emerging evidence has demonstrated that DNA repair plays a critical role in maintaining genome integrity [14]. To date, at least five known major DNA repair pathways are validated $[15,16]$, among which nucleotide excision repair (NER) is the most versatile DNA repair mechanism. It is responsible for removing bulky helix-distorting DNA lesions, such as DNA adducts caused by UV radiation, mutagenic chemicals, and chemotherapeutic agents [17]. In humans, defects in NER pathway can result in the rare autosomal recessive disease xeroderma pigmentosum $(\mathrm{XP})$, which is characterized by extreme vulnerability to UV and high susceptibility to sunlight-induced skin cancer [18]. Deficiencies in NER pathway were also associated with an increased risk of various types of cancer [19-22]. Thus far, at least seven key components (complementation groups XP-A to G) have been identified in the NER pathway [23].

Xeroderma pigmentosum group $G(X P G)$, also known as excision repair cross-complementation group 5 (ERCC5), is located on chromosome 13q22-q33, and encodes a 1,186-amino acid structure-specific endonuclease [24]. XPG is an important DNA damage recognition protein that binds to and cleaves damaged DNA at a very early stage to facilitate the downstream DNA repair process [25-27]. In addition, XPG has been implicated in RNA transcription through interaction with other transcription activator complexes [28, 29], which eventually influences mutagenesis and cell death [30]. It is widely reported that XPG is important in maintaining genomic stability and that single nucleotide polymorphisms (SNPs) in the XPG gene may affect XPG protein expression and function and contribute to DNA repair defects, genomic instability, which may leads to the initiation of cancer of various types [31,32], including CRC $[33,34]$.

A multitude of studies have been performed to investigate the association between the XPG gene polymorphisms and cancer risk [35], including lung cancer [36, 37], gastric cancer [20,38, 39], head and neck cancer [31, 40], and neuroblastoma [41]. However, regarding the association with CRC, the sample sizes in the published studies were relatively small, generally less than 1000 cases. Therefore, it is essential to precisely determine the relationship between potentially functional SNPs in XPG gene and CRC susceptibility with sufficient statistical power. Here, we conducted a two-stage case-control study to interrogate the association of interest in a Southern Chinese population consisting of 1,901 CRC patients and 1,976 healthy controls.

\section{Materials and methods}

\section{Study population}

We performed a two-stage case-control study. The first stage (discovery phase) was designed to discover the significant variants associated with CRC susceptibility in a Chinese population, consisting of 1,141 CRC cases and 1,173 cancer-free controls. The second stage (replication phase) was performed to confirm the results observed in the first-stage, consisting of $760 \mathrm{CRC}$ cases and 803 controls. The reason why we reported this study as a two-stage case control study was that the control subjects were recruited differently. Basically, 1,173 controls included in the first stage were enrolled from Sihui city, while 803 controls included in the second stage were enrolled from individuals who receiving health screening in the First Affiliated Hospital of Sun Yat-sen University. Overall, we recruited CRC cases and healthy controls mainly from January 2000 to May 2010 [42, 43]. Briefly, all the research objects were unrelated ethnic Han Chinese population from Southern China. A total of 1,901 patients with CRC were enrolled from Sun Yat-sen University Cancer Center. Cases were eligible if they had histologically confirmed adenocarcinoma of the colon; patients with metastasized cancer from other organs were excluded from the study. All the cases were sporadic CRC patients without family history of CRC, familial adenomatous polyposis syndrome, or hereditary non-polyposis colorectal cancer. All 1,976 healthy controls were randomly selected in the same region, and were frequency-matched to cases by sex. After the written informed consent was obtained from a participant, we conducted a face-to-face interview using a self-administered questionnaire including demographic characteristics (e.g., age and sex), lifestyle habits (e.g., smoking habits and alcohol drinking), as well as family history of cancer. The definition of the smoking status and drinking status has been described elsewhere [38]. With the permission of the subjects, about $5 \mathrm{ml}$ of venous blood sample was collected from each subject after interview. In general, the response rate of cases and controls was more than $80 \%$. The experimental and research protocols were approved by the Institutional Review Board of Sun Yat-sen University Cancer 
Center, and all experiments on humans samples were performed in accordance with relevant guidelines and regulations.

\section{Identification of candidate SNPs}

The potentially functional SNPs were selected as we described previously [38]. Briefly, we searched the candidate SNPs located in the $5^{\prime}$ - flanking region, exon, 5'- untranslated region (5' UTR), and 3' UTR, which might affect transcription activity and the microRNA binding site activity. The widely reported SNP rs17655 G>C was excluded because of its highly linkage disequilibrium (LD) with rs873601 G>A $\left(R^{2}=0.91\right)$. As a result, five potential functional SNPs (rs2094258 C>T, rs751402 C>T, rs2296147 $\mathrm{T}>\mathrm{C}$, rs1047768 $\mathrm{T}>\mathrm{C}$ and $\mathrm{rs} 873601 \mathrm{G}>\mathrm{A}$ ) with a minor allele frequency (MAF) $>5 \%$ for Chinese Han were selected. All of these five polymorphisms were not identified by previous genome-wide association studies. There was no significant LD among these polymorphisms $\left(\mathrm{R}^{2}<0.8\right)$. The basic parameters including location and putative function of the five selected SNPs in this study were summarized in Supplemental Table 1.

\section{Genotyping}

Genomic DNA was extracted from blood samples using the Qiagen Blood DNA Mini Kit (Qiagen Inc., Valencia, CA) following standard procedures. Genotype analyses of all SNPs were done using Taqman assays from Applied Biosystems (Foster City, CA). To obtain a high accuracy rate of genotyping results, we applied strict quality control procedures, four duplicated positive controls and four negative controls without DNA were used in each of 384-well plates. Additionally, 5\% of the samples were randomly chosen for repeatedly genotyping, and $100 \%$ concordant results were obtained for all SNPs [38].

\section{Statistical analysis}

Differences in distributions of covariates such as demographic characteristics, smoking and alcohol status, tumor sites, tumor stages, and the genotype frequency distributions of XPG polymorphism among cases and controls were compared using chi-square test. Goodness-of-fit $\chi^{2}$ test was used to test whether the genotype frequency distribution of each polymorphism in controls was in Hardy-Weinberg equilibrium (HWE). To estimate the associations between each SNP and CRC risk, univariate and multivariate logistic regression were used to calculate odds ratios (ORs) and 95\% confidence intervals (CIs). Stratification analysis by age, gender, pack-years, smoking and drinking status was performed. The genotype-based mRNA expression for the three polymorphisms (rs2094258 C>T, rs751402 C>T, and rs873601 G>A) were performed by a Student's t-test as described previously [38, 44]. All statistical analysis was performed using SAS software (version 9.1; SAS Institute, Cary, NC). All statistical tests were two-sided, and $P<0.05$ was considered significant.

\section{Results}

\section{Population characteristics}

The clinical and demographic characteristics of 1,901 colorectal cancer patients and 1,976 cancer-free controls were summarized in Table 1 . In brief, there were significant differences between the cases and controls, regarding age, smoking and alcohol status except for gender $(P=0.518)$ in the first stage. Similarly, the average age of the cases was significantly higher than that of controls, while no significant difference was seen in gender $(P=0.159)$ in the validation stage. The combined analysis of the first and second stages revealed that no significant differences in gender $(P=0.174)$ was observed between cases and controls, while patients were significantly older than controls ( $57.1 \pm 13.7$ vs. $43.6 \pm 11.9)$. Among CRC patients, $46.4 \%$ of lesions (882 cases) occurred in colon, and $53.6 \%$ of lesions $(1,019$ cases $)$ in rectum. Regarding tumor stages, 207 (10.9\%), 636 (33.5\%), 609 $(32.0 \%)$, and 449 cases (23.6\%) had Duke stage A, B, C, and $\mathrm{D}$ diseases, respectively.

\section{Associations between XPG gene polymorphisms and colorectal cancer risk}

Results were summarized in Table 2, including the allele frequency and genotype distribution of the selected XPG gene polymorphisms in CRC patients and controls, and ORs and 95\% CIs. Genotype frequency distributions of all the SNPs were in HWE within the control subjects $(P=0.623$ for rs2094258, $P=0.537$ for rs751402, $P=0.182$ for rs2296147, $P=0.875$ for rs1047768, and $P=0.875$ for rs873601). A single locus analysis showed that in the first phase, the rs2094258 C>T, rs751402 C>T, and rs873601 G>A polymorphisms were significantly associated with CRC susceptibility. The association between all these five polymorphisms and CRC risk were validated in the second stage. Interestingly, the significant associations became even more conspicuous after merging subjects from the two stages. While all subjects from two stages were combined, logistic regression analyses, after adjustment for age, gender, smoking and drinking status, revealed that the rs2094258 T allele was associated with the increased risk of CRC (Adjusted OR=1.17, 95\% CI=1.01-1.36, $P=0.043$ for $\mathrm{CT}$ vs. CC; Adjusted $\mathrm{OR}=1.49,95 \%$ $\mathrm{CI}=1.18-1.89, \quad P=0.001$ for TT vs. CC; Adjusted $\mathrm{OR}=1.38, \quad 95 \% \mathrm{CI}=1.10-1.72, \quad P=0.005$ for TT vs. 
$\mathrm{CC} / \mathrm{CT}$ ). The risk effect on CRC was also observed for the rs873601 A allele (Adjusted OR=1.18, 95\% $\mathrm{CI}=1.00-1.40, \quad P=0.055$ for AG vs. GG; Adjusted $\mathrm{OR}=1.41,95 \% \mathrm{CI}=1.15-1.72, P=0.001$ for AA vs. GG; and Adjusted $\mathrm{OR}=1.26,95 \% \mathrm{CI}=1.06-1.49, P=0.008$ for AA vs. GG/AG). On the contrary, the association in the reverse direction was found between the rs 751402 $\mathrm{T}$ allele and the risk of CRC (Adjusted OR=0.82, 95\% $\mathrm{CI}=0.70-0.96, P=0.013$ for CT vs. CC; Adjusted $\mathrm{OR}=0.69,95 \% \mathrm{CI}=0.55-0.86, P=0.001$ for TT vs. $\mathrm{CC}$; and Adjusted $\mathrm{OR}=0.77,95 \% \mathrm{CI}=0.63-0.94, P=0.011$ for TT vs. CC/CT). No significant association was observed between CRC risk and rs2296147 T>C polymorphism as well as the rs1047768 $\mathrm{T}>\mathrm{C}$ polymorphism.

\section{Stratification analysis for the three significant polymorphisms}

We further investigated the association between rs2094258 C>T, rs751402 C>T, and rs873601 G>A polymorphisms and $\mathrm{CRC}$ risk in the stratified study by age, gender, smoking status, pack-year, drinking status, tumor sites, and Duke stages. The results of the first phase were shown in Table 3. We found that the rs2094258 TT genotype was associated with a significantly increased risk of CRC risk among men
(Adjusted $\mathrm{OR}=1.54, \quad 95 \% \quad \mathrm{CI}=1.06-2.23)$, heavy cigarette smokers (Adjusted OR=2.57, 95\% $\mathrm{CI}=1.13-5.85$ ), patients with colon cancer (Adjusted $\mathrm{OR}=1.48,95 \% \mathrm{CI}=1.03-2.12$ ), and Duke stages $\mathrm{C}+\mathrm{D}$ patients (Adjusted $\mathrm{OR}=1.56,95 \% \mathrm{CI}=1.13-2.15$ ), when CC/CT genotypes were served as a reference group. Moreover, the rs873601 AA genotype was found to be associated with a significantly increased risk of CRC among women (Adjusted $\mathrm{OR}=1.47,95 \% \mathrm{CI}=1.03-2.11$ ), non-smokers (Adjusted $\mathrm{OR}=1.42,95 \% \mathrm{CI}=1.07-1.89$ ), never-drinkers (Adjusted OR=1.36, 95\% CI=1.05-1.77), patients with colon cancer (Adjusted OR=1.48, 95\% $\mathrm{CI}=1.12-1.95)$ and Duke stages $\mathrm{C}+\mathrm{D}$ diseases (Adjusted OR=1.53, 95\% CI=1.19-1.95), when GG/AG genotypes were considered as a reference group. In contrast, we found that the rs751402 TT genotype helped to protect against CRC among the young group (Adjusted $\mathrm{OR}=0.66,95 \% \mathrm{CI}=0.48-0.90$ ), men (Adjusted $\mathrm{OR}=0.65, \quad 95 \% \quad \mathrm{CI}=0.47-0.90)$, smokers (Adjusted $\mathrm{OR}=0.59,95 \% \mathrm{CI}=0.37-0.93$ ), patients with rectal cancer (Adjusted OR=0.73, 95\% CI=0.53-0.99) and Duke stages $\mathrm{C}+\mathrm{D}$ diseases (Adjusted $\mathrm{OR}=0.72$, 95\% CI=0.53-0.98), when compared with $\mathrm{CC} / \mathrm{CT}$ genotypes.

Table 1. Clinical and demographic characteristics of colorectal cancer patients and cancer-free controls.

\begin{tabular}{|c|c|c|c|c|c|c|c|c|c|}
\hline \multirow[t]{2}{*}{ Variables } & \multicolumn{3}{|l|}{ Study 1} & \multicolumn{3}{|l|}{ Study 2} & \multicolumn{3}{|l|}{ Combined } \\
\hline & Cases No. (\%) & Controls No. (\%) & $P a$ & Cases No. (\%) & Controls No. (\%) & $P a$ & Cases No. (\%) & Controls No. (\%) & $P a$ \\
\hline All subjects & $1141(100.0)$ & $1173(100.0)$ & & $760(100.0)$ & $803(100.0)$ & & $1901(100.0)$ & $1976(100.0)$ & \\
\hline \multicolumn{10}{|l|}{ Gender } \\
\hline Males & $753(66.0)$ & $789(67.3)$ & 0.518 & 397 (52.2) & $448(55.8)$ & 0.159 & $1150(60.5)$ & $1238(62.6)$ & 0.174 \\
\hline Females & $388(34.0)$ & $384(32.7)$ & & $363(47.8)$ & $355(44.2)$ & & $751(39.5)$ & $739(37.4)$ & \\
\hline Age, yr & $13-89$ & $16-80$ & & $21-91$ & $17-80$ & & $13-91$ & $16-80$ & \\
\hline Mean & $55.7 \pm 13.7$ & $45.2 \pm 11.6$ & & $59.0 \pm 13.6$ & $41.3 \pm 12.1$ & & $57.1 \pm 13.7$ & $43.6 \pm 11.9$ & \\
\hline$\leq 50$ & $367(32.2)$ & $789(67.3)$ & $<0.0001$ & $193(25.4)$ & $594(74.0)$ & $<0.0001$ & $560(29.5)$ & $1383(70.0)$ & $<0.0001$ \\
\hline $51-60$ & $342(30.0)$ & $285(24.3)$ & & $190(25.0)$ & $180(22.4)$ & & $532(28.0)$ & $466(23.6)$ & \\
\hline $61-70$ & $273(23.9)$ & $73(6.2)$ & & $205(27.0)$ & $25(3.1)$ & & $478(25.1)$ & $98(5.0)$ & \\
\hline$>70$ & 159 (13.9) & $26(2.2)$ & & $172(22.6)$ & $4(0.5)$ & & $331(17.4)$ & $30(1.5)$ & \\
\hline \multicolumn{10}{|c|}{ Smoking status } \\
\hline Never & $830(72.7)$ & $662(56.4)$ & $<0.0001$ & 566 (73.7) & - & & $1396(73.4)$ & - & \\
\hline Ever & $311(27.3)$ & $511(43.6)$ & & $194(26.3)$ & - & & 505 (26.6) & - & \\
\hline \multicolumn{10}{|c|}{ Drinking status } \\
\hline No & $968(84.8)$ & $600(51.2)$ & $<0.0001$ & $651(85.7)$ & - & & 1619 (85.2) & - & \\
\hline Yes & 173 (15.2) & $573(48.8)$ & & 109 (14.3) & - & & $282(14.8)$ & - & \\
\hline \multicolumn{10}{|l|}{ Pack-years } \\
\hline 0 & $830(72.7)$ & $662(56.4)$ & $<0.0001$ & $566(73.7)$ & - & & 1396 (73.4) & - & \\
\hline$\leq 30$ & $207(18.1)$ & $383(32.7)$ & & 138 (18.2) & - & & $345(18.2)$ & - & \\
\hline$>30$ & $104(9.1)$ & $128(10.9)$ & & $56(7.4)$ & - & & $160(8.4)$ & - & \\
\hline \multicolumn{10}{|l|}{ Tumor sites } \\
\hline Colon & $505(44.3)$ & - & & 377 (49.6) & - & & $882(46.4)$ & - & \\
\hline Rectal & $636(55.7)$ & - & & $383(50.4)$ & - & & 1019 (53.6) & - & \\
\hline \multicolumn{10}{|l|}{ Duke Stages } \\
\hline A & 130 (11.4) & - & & 77 (10.1) & - & & 207 (10.9) & - & \\
\hline B & 363 (31.8) & - & & 273 (35.9) & - & & $636(33.5)$ & - & \\
\hline C & 359 (31.5) & - & & $250(32.9)$ & - & & $609(32.0)$ & - & \\
\hline D & $289(25.3)$ & - & & $160(21.1)$ & - & & $449(23.6)$ & - & \\
\hline
\end{tabular}

a Two-sided $\chi^{2}$ test for frequency distributions between colorectal cancer cases and cancer-free controls. 
Table 2. The associations between XPG gene polymorphisms and colorectal cancer risk.

\begin{tabular}{|c|c|c|c|c|c|c|c|c|c|c|c|c|c|c|c|}
\hline & Study 1 & & & & & Study 2 & & & & & Combined & & & & \\
\hline Genotypes & Cases & Controls & $P^{a}$ & $\begin{array}{l}\text { AOR } \\
(95 \% \mathrm{CI})\end{array}$ & $P^{\mathrm{b}}$ & Cases & Controls & $P^{a}$ & $\begin{array}{l}\text { AOR } \\
(95 \% \mathrm{CI})\end{array}$ & $P^{\mathrm{b}}$ & Cases & Controls & $P a$ & $\begin{array}{l}\text { AOR } \\
(95 \% \mathrm{CI})\end{array}$ & $P^{\mathrm{b}}$ \\
\hline \multicolumn{16}{|c|}{ rs2094258 C>T } \\
\hline $\mathrm{CC}$ & $472(41.4)$ & $527(44.9)$ & & 1.00 & & $\begin{array}{l}325 \\
(42.8)\end{array}$ & $372(46.3)$ & & 1.00 & & 797 (41.9) & 899 (45.5) & & 1.00 & \\
\hline CT & $522(45.8)$ & $524(44.7)$ & & $\begin{array}{l}1.14 \\
(0.93-1.39)\end{array}$ & 0.202 & $\begin{array}{l}334 \\
(44.0)\end{array}$ & $356(44.3)$ & & $\begin{array}{l}1.22 \\
(0.94-1.58)\end{array}$ & 0.135 & $856(45.0)$ & 881 (44.6) & & $\begin{array}{l}1.17 \\
(1.01-1.36)\end{array}$ & 0.043 \\
\hline TT & 147 (12.9) & $122(10.4)$ & & $\begin{array}{l}1.44 \\
(1.06-1.95)\end{array}$ & 0.021 & $\begin{array}{l}101 \\
(13.3)\end{array}$ & $75(9.3)$ & & $\begin{array}{l}1.61 \\
(1.08-2.40)\end{array}$ & 0.020 & 248 (13.1) & $197(10.0)$ & & $\begin{array}{l}1.49 \\
(1.18-1.89)\end{array}$ & 0.001 \\
\hline Additive & & & 0.086 & $\begin{array}{l}1.18 \\
(1.03-1.35)\end{array}$ & 0.020 & & & 0.038 & $\begin{array}{l}1.25 \\
(1.05-1.50)\end{array}$ & 0.015 & & & 0.004 & $\begin{array}{l}1.20 \\
(1.08-1.34)\end{array}$ & 0.001 \\
\hline Recessive & 147 (12.9) & $122(10.4)$ & 0.063 & $\begin{array}{l}1.34 \\
(1.01-1.80)\end{array}$ & 0.046 & $\begin{array}{l}101 \\
(13.3)\end{array}$ & $75(9.3)$ & 0.014 & $\begin{array}{l}1.48 \\
(1.00-2.13)\end{array}$ & 0.051 & $248(13.1)$ & $197(10.0)$ & 0.003 & $\begin{array}{l}1.38 \\
(1.10-1.72)\end{array}$ & 0.005 \\
\hline \multicolumn{16}{|c|}{ rs751402 C>T } \\
\hline $\mathrm{CC}$ & $466(40.9)$ & $433(36.9)$ & & 1.00 & & $\begin{array}{l}326 \\
(42.9)\end{array}$ & $290(36.1)$ & & 1.00 & & 792 (41.7) & 724 (36.6) & & 1.00 & \\
\hline CT & $524(46.0)$ & $551(47.0)$ & & $\begin{array}{l}0.88 \\
(0.72-1.08)\end{array}$ & 0.221 & $\begin{array}{l}336 \\
(44.2)\end{array}$ & 401 (49.9) & & $\begin{array}{l}0.71 \\
(0.54-0.92)\end{array}$ & 0.010 & $860(45.3)$ & $952(48.2)$ & & $\begin{array}{l}0.82 \\
(0.70-0.96)\end{array}$ & 0.013 \\
\hline TT & $150(13.2)$ & $189(16.1)$ & & $\begin{array}{l}0.66 \\
(0.50-0.88)\end{array}$ & 0.005 & 98 (12.9) & $112(13.9)$ & & $\begin{array}{l}0.63 \\
(0.43-0.92)\end{array}$ & 0.018 & $248(13.1)$ & 301 (15.2) & & $\begin{array}{l}0.69 \\
(0.55-0.86)\end{array}$ & 0.001 \\
\hline Additive & & & 0.052 & $\begin{array}{l}0.83 \\
(0.73-0.95)\end{array}$ & 0.006 & & & 0.023 & $\begin{array}{l}0.77 \\
(0.64-0.92)\end{array}$ & 0.004 & & & 0.004 & $\begin{array}{l}0.83 \\
(0.75-0.92)\end{array}$ & 0.0004 \\
\hline Recessive & $150(13.2)$ & $189(16.1)$ & 0.045 & $\begin{array}{l}0.71 \\
(0.55-0.92)\end{array}$ & 0.010 & 98 (12.9) & $112(13.9)$ & 0.542 & $\begin{array}{l}0.76 \\
(0.53-1.08)\end{array}$ & 0.128 & 248 (13.1) & 301 (15.2) & 0.052 & $\begin{array}{l}0.77 \\
(0.63-0.94)\end{array}$ & 0.011 \\
\hline \multicolumn{16}{|c|}{ rs2296147 T>C } \\
\hline TT & $714(62.6)$ & $746(63.6)$ & & 1.00 & & $\begin{array}{l}455 \\
(59.9)\end{array}$ & $467(58.2)$ & & 1.00 & & 1169 (61.5) & $1213(61.4)$ & & 1.00 & \\
\hline CT & $379(33.2)$ & $388(33.1)$ & & $\begin{array}{l}1.12 \\
(0.92-1.37)\end{array}$ & 0.260 & $\begin{array}{l}265 \\
(34.9)\end{array}$ & $303(37.7)$ & & $\begin{array}{l}0.93 \\
(0.72-1.21)\end{array}$ & 0.595 & $644(33.9)$ & $692(35.0)$ & & $\begin{array}{l}1.01 \\
(0.86-1.17)\end{array}$ & 0.951 \\
\hline $\mathrm{CC}$ & $48(4.2)$ & $39(3.3)$ & & $\begin{array}{l}1.33 \\
(0.81-2.17)\end{array}$ & 0.264 & $40(5.3)$ & $33(4.1)$ & & $\begin{array}{l}1.66 \\
(0.90-3.05)\end{array}$ & 0.102 & $88(4.6)$ & $72(3.6)$ & & $\begin{array}{l}1.34 \\
(0.92-1.93)\end{array}$ & 0.124 \\
\hline Additive & & & 0.523 & $\begin{array}{l}1.13 \\
(0.96-1.34)\end{array}$ & 0.140 & & & 0.335 & $\begin{array}{l}1.06 \\
(0.86-1.31)\end{array}$ & 0.568 & & & 0.266 & $\begin{array}{l}1.06 \\
(0.94-1.20)\end{array}$ & 0.353 \\
\hline Recessive & $48(4.2)$ & $39(3.3)$ & 0.265 & $\begin{array}{l}1.27 \\
(0.78-2.08)\end{array}$ & 0.332 & $40(5.3)$ & $33(4.1)$ & 0.280 & $\begin{array}{l}1.71 \\
(0.93-3.11)\end{array}$ & 0.082 & $88(4.6)$ & $72(3.6)$ & 0.122 & $\begin{array}{l}1.33 \\
(0.93-1.92)\end{array}$ & 0.122 \\
\hline \multicolumn{16}{|c|}{ rs1047768 T>C } \\
\hline TT & $592(51.9)$ & $625(53.3)$ & & 1.00 & & $\begin{array}{l}378 \\
(49.7)\end{array}$ & $398(49.6)$ & & 1.00 & & $970(51.0)$ & $1023(51.7)$ & & 1.00 & \\
\hline TC & 451 (39.5) & 461 (39.3) & & $\begin{array}{l}1.09 \\
(0.89-1.32)\end{array}$ & 0.415 & $\begin{array}{l}307 \\
(40.4)\end{array}$ & 350 (43.6) & & $\begin{array}{l}0.99 \\
(0.77-1.27)\end{array}$ & 0.913 & 758 (39.9) & 812 (41.1) & & $\begin{array}{l}1.00 \\
(0.86-1.16)\end{array}$ & 0.960 \\
\hline $\mathrm{CC}$ & $98(8.6)$ & $87(7.4)$ & & $\begin{array}{l}1.22 \\
(0.86-1.74)\end{array}$ & 0.267 & $75(9.9)$ & $55(6.8)$ & & $\begin{array}{l}1.75 \\
(1.09-2.81)\end{array}$ & 0.020 & $173(9.1)$ & $142(7.2)$ & & $\begin{array}{l}1.33 \\
(1.01-1.75)\end{array}$ & 0.039 \\
\hline Additive & & & 0.544 & $\begin{array}{l}1.10 \\
(0.95-1.27)\end{array}$ & 0.217 & & & 0.073 & $\begin{array}{l}1.16 \\
(0.96-1.41)\end{array}$ & 0.132 & & & 0.089 & $\begin{array}{l}1.08 \\
(0.97-1.21)\end{array}$ & 0.169 \\
\hline Recessive & $98(8.6)$ & $87(7.4)$ & 0.299 & $\begin{array}{l}1.18 \\
(0.84-1.66)\end{array}$ & 0.346 & $75(9.9)$ & $55(6.8)$ & 0.031 & $\begin{array}{l}1.76 \\
(1.11-2.79)\end{array}$ & 0.016 & $173(9.1)$ & $142(7.2)$ & 0.029 & $\begin{array}{l}1.34 \\
(1.02-1.74)\end{array}$ & 0.033 \\
\hline \multicolumn{16}{|c|}{ rs873601 G>A } \\
\hline GG & $266(23.3)$ & $323(27.5)$ & & 1.00 & & $\begin{array}{l}210 \\
(27.6)\end{array}$ & $227(28.3)$ & & 1.00 & & $476(25.0)$ & $550(27.8)$ & & 1.00 & \\
\hline AG & $579(50.7)$ & $598(51.0)$ & & $\begin{array}{l}1.20 \\
(0.96-1.50)\end{array}$ & 0.112 & $\begin{array}{l}375 \\
(49.3)\end{array}$ & $426(53.1)$ & & $\begin{array}{l}1.17 \\
(0.87-1.56)\end{array}$ & 0.294 & $954(50.2)$ & 1025 (51.9) & & $\begin{array}{l}1.18 \\
(1.00-1.40)\end{array}$ & 0.055 \\
\hline AA & $296(25.9)$ & $252(21.5)$ & & $\begin{array}{l}1.49 \\
(1.14-1.94)\end{array}$ & 0.003 & $\begin{array}{l}175 \\
(23.0)\end{array}$ & 150 (18.7) & & $\begin{array}{l}1.29 \\
(0.91-1.83)\end{array}$ & 0.147 & $471(24.8)$ & 402 (20.3) & & $\begin{array}{l}1.41 \\
(1.15-1.72)\end{array}$ & 0.001 \\
\hline Additive & & & 0.012 & $\begin{array}{l}1.22 \\
(1.07-1.39)\end{array}$ & 0.004 & & & 0.098 & $\begin{array}{l}1.14 \\
(0.96-1.36)\end{array}$ & 0.143 & & & 0.003 & $\begin{array}{l}1.19 \\
(1.07-1.31)\end{array}$ & 0.001 \\
\hline Recessive & $296(25.9)$ & $252(21.5)$ & 0.012 & $\begin{array}{l}1.32 \\
(1.06-1.64)\end{array}$ & 0.014 & $\begin{array}{l}175 \\
(23.0)\end{array}$ & $150(18.7)$ & 0.034 & $\begin{array}{l}1.17 \\
(0.87-1.56)\end{array}$ & 0.295 & $471(24.8)$ & $402(20.3)$ & 0.001 & $\begin{array}{l}1.26 \\
(1.06-1.49)\end{array}$ & 0.008 \\
\hline
\end{tabular}

XPG, xeroderma pigmentosum group G; CI, confidence interval; AOR, adjusted odds ratio.

a $\chi^{2}$ test for genotype distributions between cases and controls.

$\mathrm{b}$ Adjusted for age, gender, smoking and drinking status in logistic regression models.

Table 3. Stratification analysis for associations between the three XPG variant genotypes and colorectal cancer risk (Study 1).

\begin{tabular}{|c|c|c|c|c|c|c|c|c|c|c|c|c|}
\hline \multirow[t]{2}{*}{ Variables } & \multicolumn{2}{|c|}{$\begin{array}{l}\text { rs2094258 } \\
\text { (cases/controls) }\end{array}$} & \multirow[t]{2}{*}{$\begin{array}{l}\text { Adjusted OR } \\
(95 \% \mathrm{CI})\end{array}$} & \multirow[t]{2}{*}{$P$ a } & \multicolumn{2}{|c|}{$\begin{array}{l}\text { rs751402 } \\
\text { (cases/controls) }\end{array}$} & \multirow[t]{2}{*}{$\begin{array}{l}\text { Adjusted OR } \\
(95 \% \mathrm{CI})\end{array}$} & \multirow[t]{2}{*}{$P$ a } & \multicolumn{2}{|c|}{ rs873601 (Case/Control) } & \multirow[t]{2}{*}{$\begin{array}{l}\text { Adjusted OR } \\
(95 \% \mathrm{CI})\end{array}$} & \multirow[t]{2}{*}{$P$ a } \\
\hline & $\mathrm{CC} / \mathrm{CT}$ & TT & & & $\mathrm{CC} / \mathrm{CT}$ & TT & & & GG/AG & $\mathrm{AA}$ & & \\
\hline \multicolumn{13}{|c|}{ Median age, yr } \\
\hline$\leq$ Median & $520 / 891$ & $78 / 108$ & $1.18(0.85-1.65)$ & 0.319 & $524 / 836$ & $74 / 163$ & $0.66(0.48-0.90)$ & 0.009 & $444 / 782$ & $154 / 217$ & $1.26(0.98-1.62)$ & 0.074 \\
\hline$>$ Median & $474 / 160$ & $69 / 14$ & $1.80(0.97-3.32)$ & 0.061 & $466 / 148$ & $76 / 26$ & $0.92(0.56-1.50)$ & 0.723 & $401 / 139$ & $142 / 35$ & $1.45(0.94-2.21)$ & 0.090 \\
\hline \multicolumn{13}{|l|}{ Gender } \\
\hline Males & $660 / 712$ & $93 / 77$ & $1.54(1.06-2.23)$ & 0.024 & $653 / 655$ & $99 / 134$ & $0.65(0.47-0.90)$ & 0.009 & $576 / 625$ & $177 / 164$ & $1.22(0.92-1.62)$ & 0.167 \\
\hline Females & $334 / 339$ & $54 / 45$ & $1.06(0.66-1.68)$ & 0.817 & $337 / 329$ & $51 / 55$ & $0.84(0.54-1.33)$ & 0.463 & $269 / 296$ & $119 / 88$ & $1.47(1.03-2.11)$ & 0.036 \\
\hline \multicolumn{13}{|c|}{ Smoking status } \\
\hline
\end{tabular}




\begin{tabular}{|c|c|c|c|c|c|c|c|c|c|c|c|c|}
\hline \multirow[t]{2}{*}{ Variables } & \multicolumn{2}{|c|}{$\begin{array}{l}\text { rs2094258 } \\
\text { (cases/controls) }\end{array}$} & \multirow[t]{2}{*}{$\begin{array}{l}\text { Adjusted OR } \\
(95 \% \mathrm{CI})\end{array}$} & \multirow[t]{2}{*}{$P$ a } & \multicolumn{2}{|c|}{$\begin{array}{l}\text { rs751402 } \\
\text { (cases/controls) }\end{array}$} & \multirow[t]{2}{*}{$\begin{array}{l}\text { Adjusted OR } \\
(95 \% \mathrm{CI})\end{array}$} & \multirow[t]{2}{*}{$P$ a } & \multicolumn{2}{|c|}{ rs873601 (Case/Control) } & \multirow[t]{2}{*}{$\begin{array}{l}\text { Adjusted OR } \\
(95 \% \mathrm{CI})\end{array}$} & \multirow[t]{2}{*}{$P$ a } \\
\hline & $\mathrm{CC} / \mathrm{CT}$ & TT & & & $\mathrm{CC} / \mathrm{CT}$ & TT & & & GG/AG & AA & & \\
\hline Never & $727 / 594$ & $103 / 68$ & $1.24(0.85-1.81)$ & 0.259 & $715 / 559$ & $114 / 103$ & $0.76(0.54-1.06)$ & 0.105 & $615 / 521$ & $215 / 141$ & $1.42(1.07-1.89)$ & 0.016 \\
\hline Ever & $267 / 457$ & $44 / 54$ & $1.60(1.00-2.56)$ & 0.049 & $275 / 425$ & $36 / 86$ & $0.59(0.37-0.93)$ & 0.023 & $230 / 400$ & $81 / 111$ & $1.23(0.85-1.77)$ & 0.277 \\
\hline \multicolumn{13}{|l|}{ Pack-years } \\
\hline 0 & $727 / 594$ & $103 / 68$ & $1.24(0.85-1.81)$ & 0.259 & $715 / 559$ & $114 / 103$ & $0.76(0.54-1.06)$ & 0.105 & $615 / 521$ & $215 / 141$ & $1.42(1.07-1.89)$ & 0.016 \\
\hline$\leq 30$ & $181 / 343$ & $26 / 40$ & $1.23(0.68-2.20)$ & 0.497 & $181 / 322$ & $26 / 61$ & $0.67(0.38-1.18)$ & 0.167 & $152 / 295$ & $55 / 88$ & $1.14(0.72-1.78)$ & 0.581 \\
\hline$>30$ & $86 / 114$ & $18 / 14$ & $2.57(1.13-5.85)$ & 0.024 & $94 / 103$ & $10 / 25$ & $0.46(0.21-1.04)$ & 0.063 & $78 / 105$ & $26 / 23$ & $1.43(0.73-2.77)$ & 0.296 \\
\hline \multicolumn{13}{|c|}{ Drinking status } \\
\hline Never & $845 / 534$ & $123 / 66$ & $1.30(0.92-1.82)$ & 0.137 & $834 / 499$ & $133 / 101$ & $0.74(0.55-1.00)$ & 0.052 & $715 / 470$ & $253 / 130$ & $1.36(1.05-1.77)$ & 0.019 \\
\hline Ever & $149 / 517$ & $24 / 56$ & $1.42(0.78-2.57)$ & 0.248 & $156 / 485$ & $17 / 88$ & $0.55(0.29-1.04)$ & 0.067 & $130 / 451$ & $43 / 122$ & $1.26(0.78-2.02)$ & 0.343 \\
\hline \multicolumn{13}{|c|}{ Tumor sites } \\
\hline Colon & $436 / 1051$ & $69 / 122$ & $1.48(1.03-2.12)$ & 0.034 & $440 / 984$ & $65 / 189$ & $0.72(0.51-1.01)$ & 0.057 & $368 / 921$ & $137 / 252$ & $1.48(1.12-1.95)$ & 0.006 \\
\hline Rectal & $558 / 1051$ & $78 / 122$ & $1.28(0.91-1.79)$ & 0.155 & $550 / 984$ & $85 / 189$ & $0.73(0.53-0.99)$ & 0.042 & $477 / 921$ & $159 / 252$ & $1.18(0.92-1.53)$ & 0.197 \\
\hline \multicolumn{13}{|c|}{ Duke stages } \\
\hline $\mathrm{A}+\mathrm{B}$ & $439 / 1051$ & $54 / 122$ & $1.06(0.72-1.58)$ & 0.762 & $427 / 984$ & $65 / 189$ & $0.72(0.51-1.03)$ & 0.068 & $379 / 921$ & $114 / 252$ & $1.01(0.76-1.36)$ & 0.928 \\
\hline$C+D$ & $555 / 1051$ & $93 / 122$ & $1.56(1.13-2.15)$ & 0.007 & $563 / 984$ & $85 / 189$ & $0.72(0.53-0.98)$ & 0.035 & $466 / 921$ & $182 / 252$ & 1.53 (1.19-1.95) & 0.001 \\
\hline
\end{tabular}

XPG, xeroderma pigmentosum group G; CI, confidence interval; OR, odds ratio.

a Adjusted for age, gender, smoking and drinking status in logistic regression models.

Table 4. Stratification analysis for associations between the three XPG variant genotypes and colorectal cancer risk (Combined).

\begin{tabular}{|c|c|c|c|c|c|c|c|c|c|c|c|c|}
\hline \multirow[t]{2}{*}{ Variables } & \multicolumn{2}{|c|}{ rs2094258 (cases/controls) } & \multirow{2}{*}{$\begin{array}{l}\text { Adjusted OR } \\
(95 \% \mathrm{CI})\end{array}$} & \multirow[t]{2}{*}{$P$ a } & \multicolumn{2}{|c|}{ rs751402 (cases/controls) } & \multirow{2}{*}{$\begin{array}{l}\text { Adjusted OR } \\
(95 \% \mathrm{CI})\end{array}$} & \multirow[t]{2}{*}{$P$ a } & \multicolumn{2}{|c|}{ rs873601 (Case/Control) } & \multirow{2}{*}{$\begin{array}{l}\text { Adjusted OR } \\
(95 \% \mathrm{CI})\end{array}$} & \multirow[t]{2}{*}{$P$ a } \\
\hline & $\mathrm{CC} / \mathrm{CT}$ & TT & & & $\mathrm{CC}$ & $\mathrm{CT} / \mathrm{TT}$ & & & GG/AG & AA & & \\
\hline \multicolumn{13}{|c|}{ Median age, yr } \\
\hline$\leq$ Median & $854 / 1576$ & $131 / 180$ & $1.34(1.05-1.70)$ & 0.017 & $422 / 640$ & $563 / 1116$ & $0.77(0.65-0.90)$ & 0.001 & $731 / 1399$ & $254 / 357$ & 1.35 (1.13-1.63) & 0.001 \\
\hline$>$ Median & $799 / 204$ & $117 / 17$ & $1.75(1.03-2.98)$ & 0.039 & $370 / 84$ & $545 / 137$ & $0.90(0.67-1.22)$ & 0.513 & $699 / 176$ & $217 / 45$ & $1.21(0.85-1.74)$ & 0.294 \\
\hline \multicolumn{13}{|l|}{ Gender } \\
\hline Males & $1005 / 1119$ & $145 / 119$ & $1.41(1.06-1.88)$ & 0.018 & $474 / 449$ & $675 / 789$ & $0.78(0.65-0.94)$ & 0.010 & $879 / 993$ & $271 / 245$ & $1.20(0.97-1.50)$ & 0.100 \\
\hline Females & $648 / 661$ & $103 / 78$ & $1.32(0.93-1.88)$ & 0.124 & $318 / 275$ & $433 / 464$ & $0.80(0.64-1.02)$ & 0.067 & $551 / 582$ & $200 / 157$ & $1.34(1.03-1.76)$ & 0.031 \\
\hline \multicolumn{13}{|c|}{ Tumor sites } \\
\hline Colon & $775 / 1780$ & $107 / 197$ & $1.40(1.06-1.85)$ & 0.020 & $362 / 724$ & $520 / 1253$ & $0.80(0.67-0.97)$ & 0.020 & $666 / 1575$ & $216 / 402$ & 1.29 (1.04-1.59) & 0.020 \\
\hline Rectal & $878 / 1780$ & $141 / 197$ & $1.38(1.06-1.79)$ & 0.015 & $430 / 724$ & $588 / 1253$ & $0.81(0.68-0.96)$ & 0.016 & $764 / 1575$ & $255 / 402$ & $1.19(0.97-1.45)$ & 0.094 \\
\hline \multicolumn{13}{|c|}{ Duke stages } \\
\hline $\mathrm{A}+\mathrm{B}$ & $736 / 1780$ & $107 / 197$ & $1.26(0.94-1.69)$ & 0.123 & $344 / 724$ & $498 / 1253$ & $0.81(0.67-0.98)$ & 0.033 & $647 / 1575$ & $196 / 402$ & $1.03(0.82-1.29)$ & 0.825 \\
\hline$C+D$ & $917 / 1780$ & $141 / 197$ & 1.47 (1.14-1.90) & 0.003 & $448 / 724$ & $610 / 1253$ & $0.80(0.68-0.95)$ & 0.009 & $783 / 1575$ & $275 / 402$ & 1.39 (1.15-1.69) & 0.001 \\
\hline
\end{tabular}

XPG, xeroderma pigmentosum group G; CI, confidence interval; OR, odds ratio.

a Adjusted for age, gender, smoking and drinking status in logistic regression models.

To further validate the association of the above SNPs with predisposition to CRC, we carried out the stratified analyses of rs2094258 C $>\mathrm{T}$, rs751402 C>T, and $\mathrm{rs} 873601 \mathrm{G}>\mathrm{A}$, using the combined sample (Table 4). With the increased number of sample, we found that the significant association with the rs2094258 TT genotype became stronger in men and patients with Duke stages $\mathrm{C}+\mathrm{D}$ diseases, and became weaker in subgroups divided by age and tumor site, when CC/CT genotypes were used as a reference group. The protective effect of rs751402 TT genotype became stronger in the young group and men, and was weakened in subgroups divided by tumor stage and tumor site. Finally, the risk effect of the rs873601 AA genotype was more evident in the young group, women, colon cancer, and Duke stages C+D subgroups, when GG/AG were used as control genotypes.

The correlation between genotypes of XPG polymorphisms and mRNA expression were shown in Supplemental Table 2. We found significant correlation between alterations in the mRNA expression and all the three noteworthy polymorphisms, to different extents.

\section{Discussion}

In the present study, we performed a two-stage case-control study to investigate the associations between five potentially functional SNPs in the XPG gene and CRC susceptibility in a Chinese Han population from Southern China. We identified three SNPs (rs2094258 C>T, rs751402 C>T and rs873601 G>A) that were associated with CRC susceptibility (first stage), and further validated these findings in a separate case-control study (second study). To the best of our knowledge, by far, the present study is the largest investigation to explore the association of XPG gene polymorphisms with CRC risk.

XPG acts as an endonuclease to participate in two incision steps in NER pathway, dysfunction of which leads to the excision repair deficiency $[45,46]$. XPG cleaves the DNA strand at the 3 ' side of the damaged site, and stabilizes the DNA repair complex 
to the damaged DNA with ERCC1/XPF complex by making $5^{\prime}$ incision [47-50]. Many XPG gene polymorphisms can change the ability of the encoded enzyme to repair the DNA damage. Genetic variants may modulate susceptibility to colorectal cancer through complex gene-gene and gene-environment interactions [51, 52].

Although a few studies have investigated the role of XPG gene polymorphisms in the CRC risk, most of them were focused on XPG Asp1104His (rs17655 G>C) polymorphism. In a hospital-based case-control study consisting of 532 cases and 532 controls from the Czech population, a trend towards an increased risk of CRC was found among carriers of XPG Asp1104His variant alleles [53]. Mort et al. [54] reported that no significant association was found between XPG Asp1104His and CRC risk. Such null association was also observed in 79 CRC patients and 247 healthy controls in Turkish population [55]. In contrast, other studies reported opposite results regarding the association. In a Poland case-control study including 758 patients with CRC and 1,841 healthy controls, the XPG Asp1104His was significantly associated with an increased CRC risk in heterozygotes (GC genotypes) [34]. Liu et al. [56] found that heterozygotes and variant homozygotes of XPG Asp1104His had an increased risk for developing CRC in a population-based case-control study involving 1,028 CRC cases and 1,085 controls in the Chinese population. And the same study indicated that this SNP also conferred a significantly decreased progression-free survival after oxaliplatin-based adjuvant chemotherapy in CRC patients. More recently, Du et al. [57] found that XPG Asp1104His polymorphism was associated with a significantly increased CRC risk, especially in Asians.

In this study, we chose four SNPs (rs2094258 $\mathrm{C}>\mathrm{T}$, rs751402 $\mathrm{C}>\mathrm{T}$, rs2296147 $\mathrm{T}>\mathrm{C}$, and rs873601 $\mathrm{G}>\mathrm{A})$ located at the two ends of the XPG gene, and one SNP (rs1047768 T>C) in the coding region. Overall, rs2094258 C>T and rs873601 G>A were shown to significantly increase CRC risk, whereas rs751402 C>T significantly reduce the CRC risk. In a previous study, we also found that the presence of rs873601A variant genotypes was associated with a significantly elevated risk of gastric cancer in an Eastern Chinese population [38]. Stratification analysis of participants in the two stages suggested that male carriers of rs2094258 TT genotype showed increased CRC risk and CRC patients carrying the rs2094258 TT genotype was significantly more likely to be diagnosed with the late stages of disease (Dukes $C+D)$. We also found that rs751402 TT genotype had a protective effect against $\mathrm{CRC}$ in the younger subjects and men. Finally, significant associations between rs873601 AA genotype and increased CRC risk were observed in the young group and women; moreover, subjects with rs873601 AA genotype tended to develop colon cancer and have Duke stages C/D diseases. In order to provide biological evidence for our findings from observational study, we performed genotype-based mRNA expression analysis using the HapMap data and found that all of these three significant polymorphisms can alter the XPG mRNA expression, to some extent. However, the precise mechanism for the association of $2094258 \mathrm{C}>\mathrm{T}$, rs751402 C>T, and rs873601 G>A polymorphisms with CRC remains unclear, because of lacking of direct functional data for these polymorphisms. Our mechanistic study based on bioinformatics analyses was informative, but quite preliminary. Besides, CRC is a complex condition caused by the interplay of environmental and genetic factors. Therefore, additional mechanistic studies are needed to unravel the molecular mechanisms underlying these observed associations.

Although we extensively analyzed five potentially functional SNPs in the XPG gene by adopting a large two-stage case-control study, followed by genotype-based mRNA expression analysis, there still exists some limitations to be addressed. First, frequency matching between cases and controls was only performed on gender, but not on age ( \pm 5 year), smoking and drinking status. Multivariate logistic regression analysis was used to reduce the impact of these factors, to some extent. Second, the number of the selected XPG polymorphisms is limited, because this research focused on the potentially functional SNPs loci not covered by the GWAS chip analysis. As a result, some nonfunctional, low-frequency SNPs and the chain with the selected sites SNPs loci, which may also contribute to CRC susceptibility, were not included in this study. For instance, rs17655 G>C, which was in strong LD with rs873601 G>A, was not selected. The selected sites were also limited, which restricted further haplotypes analysis. Third, the inherent limitation of retrospective study, to a certain extent, reduced the credibility of the assessment of the CRC risk. Many other related factors such as BMI, eating habits, occupational exposure and environmental factors should be considered, since colorectal cancer is a heterogeneous disease. Forth, our study was a case-control study with subjects from Southern China, and this population may not well represent other Chinese populations in the different regions. Finally, in this hospital-based case-control study, patients were recruited from hospitals while controls were from the community. As a result, selection bias and information bias may exist. The phenomenon that the 
controls were more likely to be smokers and drinkers compared to the CRC group in our study may be the results of selection bias. Moreover, it should be noted that the mean age of the CRC patients was quite low in this study.

In conclusion, we found the XPG rs $2094258 \mathrm{C}>\mathrm{T}$, rs751402 $\mathrm{C}>\mathrm{T}$ and rs873601 $\mathrm{G}>\mathrm{A}$ polymorphisms were associated with CRC susceptibility. Future well-designed, prospective studies with larger sample size, involving different ethnicities, are warranted to confirm our findings.

\section{Supplementary Material}

Supplementary tables.

http://www.jcancer.org/v07p1731s1.pdf

\section{Abbreviations}

CRC, colorectal cancer; NER, nucleotide excision repair; $\mathrm{XP}$, xeroderma pigmentosum; $\mathrm{XPG}$, xeroderma pigmentosum group G; ERCC5, excision repair cross-complementation group 5; SNP, single nucleotide polymorphisms; MAF, minor allele frequency; HWE, Hardy-Weinberg equilibrium; OR, odds ratio; $\mathrm{CI}$, confidence interval; $\mathrm{LD}$, linkage disequilibrium.

\section{Acknowledgement}

This study was supported by grants from the National Natural Science Foundation of China (Grant No. 81502046), Special Financial Grant from the China Postdoctoral Science Foundation (Grant No. 2014T70836), the Natural Science Foundation of Guangdong Province (Grant No. 2015A030310324), and the National Science Fund for Distinguished Young Scholars (Grant No. 81325018).

\section{Conflict of interest}

The authors declare no conflicts of interest.

\section{References}

1. Torre LA, Bray F, Siegel RL, Ferlay J, Lortet-Tieulent J, Jemal A. Global cancer statistics, 2012. CA Cancer J Clin. 2015; 65: 87-108.

2. Center MM, Jemal A, Smith RA, Ward E. Worldwide variations in colorectal cancer. CA Cancer J Clin. 2009; 59: 366-78.

3. Zhao P, Dai M, Chen W, Li N. Cancer trends in China. Jpn J Clin Oncol. 2010; 40: $281-5$.

4. Fahy B, Bold RJ. Epidemiology and molecular genetics of colorectal cancer. Surg Oncol. 1998; 7: 115-23.

5. Brenner H, Kloor M, Pox CP. Colorectal cancer. Lancet. 2014; 383: 1490-502.

6. Ferrari P, Jenab M, Norat T, Moskal A, Slimani N, Olsen A, et al. Lifetime and baseline alcohol intake and risk of colon and rectal cancers in the European prospective investigation into cancer and nutrition (EPIC). Int J Cancer. 2007; 121: 2065-72.

7. Gong J, Tian J, Lou J, Ke J, Li L, Li J, et al. A functional polymorphism in Inc-LAMC2-1:1 confers risk of colorectal cancer by affecting miRNA binding. Carcinogenesis. 2016; 37: 443-51.

8. Ke J, Lou J, Chen X, Li J, Liu C, Gong Y, et al. Identification of a functional variant for colorectal cancer risk mapping to chromosome 5q31.1. Oncotarget. 2016. DOI:10.18632/oncotarget.9298.

9. Zhang B, Jia WH, Matsuo K, Shin A, Xiang YB, Matsuda K, et al. Genome-wide association study identifies a new SMAD7 risk variant associated with colorectal cancer risk in East Asians. Int J Cancer. 2014; 135: 948-55.
10. Wang M, Gu D, Du M, Xu Z, Zhang S, Zhu L, et al. Common genetic variation in ETV6 is associated with colorectal cancer susceptibility. Nat Commun. 2016; 7: 11478 .

11. Zeng C, Matsuda K, Jia WH, Chang J, Kweon SS, Xiang YB, et al. Identification of Susceptibility Loci and Genes for Colorectal Cancer Risk. Gastroenterology. 2016. DOI: 10.1053/j.gastro.2016.02.076.

12. Cui R, Okada Y, Jang SG, Ku JL, Park JG, Kamatani Y, et al. Common variant in 6q26-q27 is associated with distal colon cancer in an Asian population. Gut. 2011; 60: 799-805.

13. Zhang B, Jia WH, Matsuda K, Kweon SS, Matsuo K, Xiang YB, et al. Large-scale genetic study in East Asians identifies six new loci associated with colorectal cancer risk. Nat Genet. 2014; 46: 533-42.

14. Ishikawa T, Zhang SS, Qin X, Takahashi Y, Oda H, Nakatsuru Y, et al. DNA repair and cancer: lessons from mutant mouse models. Cancer Sci. 2004; 95: 112-7.

15. Hoeijmakers JH. Genome maintenance mechanisms for preventing cancer. Nature. 2001; 411: 366-74.

16. Wood RD, Mitchell M, Sgouros J, Lindahl T. Human DNA repair genes. Science. 2001; 291: 1284-9.

17. Leibeling D, Laspe P, Emmert S. Nucleotide excision repair and cancer. J Mol Histol. 2006; 37: 225-38

18. Constantinou A, Gunz D, Evans E, Lalle P, Bates PA, Wood RD, et al. Conserved residues of human XPG protein important for nuclease activity and function in nucleotide excision repair. J Biol Chem. 1999; 274: 5637-48.

19. Berhane N, Sobti RC, Mahdi SA. DNA repair genes polymorphism (XPG and $\mathrm{XRCC} 1$ ) and association of prostate cancer in a north Indian population. Mol Biol Rep. 2012; 39: 2471-9.

20. Li Y, Liu Z, Liu H, Wang LE, Onodera H, Suzuki A, et al. Potentially functional variants in the core nucleotide excision repair genes predict survival in Japanese gastric cancer patients. Carcinogenesis. 2014; 35: 2031-8.

21. Negandhi AA, Hyde A, Dicks E, Pollett W, Younghusband BH, Parfrey P, et al. MTHFR Glu429Ala and ERCC5 His46His polymorphisms are associated with prognosis in colorectal cancer patients: analysis of two independent cohorts from Newfoundland. PLoS One. 2013; 8: e61469.

22. Jung SW, Park NH, Shin JW, Park BR, Kim CJ, Lee JE, et al. Polymorphisms of DNA repair genes in Korean hepatocellular carcinoma patients with chronic hepatitis B: possible implications on survival. J Hepatol. 2012; 57: 621-7.

23. De Weerd-Kastelein EA, Keijzer W, Bootsma D. Genetic heterogeneity of xeroderma pigmentosum demonstrated by somatic cell hybridization. Nat New Biol. 1972; 238: 80-3.

24. Emmert S, Schneider TD, Khan SG, Kraemer KH. The human XPG gene: gene architecture, alternative splicing and single nucleotide polymorphisms. Nucleic Acids Res. 2001; 29: 1443-52.

25. Gillet LC, Scharer OD. Molecular mechanisms of mammalian global genome nucleotide excision repair. Chem Rev. 2006; 106: 253-76.

26. Lin J, Swan GE, Shields PG, Benowitz NL, Gu J, Amos CI, et al. Mutagen sensitivity and genetic variants in nucleotide excision repair pathway: genotype-phenotype correlation. Cancer Epidemiol Biomarkers Prev. 2007; 16: 2065-71.

27. Scherly D, Nouspikel T, Corlet J, Ucla C, Bairoch A, Clarkson SG. Complementation of the DNA repair defect in xeroderma pigmentosum group G cells by a human cDNA related to yeast RAD2. Nature. 1993; 363: 182-5.

28. Lee SK, Yu SL, Prakash L, Prakash S. Requirement of yeast RAD2, a homolog of human XPG gene, for efficient RNA polymerase II transcription. implications for Cockayne syndrome. Cell. 2002; 109: 823-34

29. Barreto G, Schafer A, Marhold J, Stach D, Swaminathan SK, Handa V, et al. Gadd45a promotes epigenetic gene activation by repair-mediated DNA demethylation. Nature. 2007; 445: 671-5.

30. Wang JY, Sarker AH, Cooper PK, Volkert MR. The single-strand DNA binding activity of human PC4 prevents mutagenesis and killing by oxidative DNA damage. Mol Cell Biol. 2004; 24: 6084-93.

31. Cheng L, Sturgis EM, Eicher SA, Spitz MR, Wei Q. Expression of nucleotide excision repair genes and the risk for squamous cell carcinoma of the head and neck. Cancer. 2002; 94: 393-7.

32. Koeppel F, Poindessous V, Lazar V, Raymond E, Sarasin A, Larsen AK. Irofulven cytotoxicity depends on transcription-coupled nucleotide excision repair and is correlated with XPG expression in solid tumor cells. Clin Cancer Res. 2004; 10: 5604-13.

33. Moreno V, Gemignani F, Landi S, Gioia-Patricola L, Chabrier A, Blanco I, et al. Polymorphisms in genes of nucleotide and base excision repair: risk and prognosis of colorectal cancer. Clin Cancer Res. 2006; 12: 2101-8.

34. Paszkowska-Szczur K, Scott RJ, Gorski B, Cybulski C, Kurzawski G, Dymerska $\mathrm{D}$, et al. Polymorphisms in nucleotide excision repair genes and susceptibility to colorectal cancer in the Polish population. Mol Biol Rep. 2015; 42: 755-64.

35. Zhu ML, Shi TY, Hu HC, He J, Wang M, Jin L, et al. Polymorphisms in the ERCC5 gene and risk of esophageal squamous cell carcinoma (ESCC) in Eastern Chinese populations. PLoS One. 2012; 7: e41500.

36. Sun X, Li F, Sun N, Shukui Q, Baoan C, Jifeng F, et al. Polymorphisms in XRCC1 and XPG and response to platinum-based chemotherapy in advanced non-small cell lung cancer patients. Lung Cancer. 2009; 65: 230-6.

37. Massuti B, Cobo M, Camps C, Domine M, Provencio M, Alberola V, et al. Trabectedin in patients with advanced non-small-cell lung cancer (NSCLC) with XPG and/or ERCC1 overexpression and BRCA1 underexpression and pretreated with platinum. Lung Cancer. 2012; 76: 354-61. 
38. He J, Qiu LX, Wang MY, Hua RX, Zhang RX, Yu HP, et al. Polymorphisms in the XPG gene and risk of gastric cancer in Chinese populations. Hum Genet. 2012; 131: 1235-44

39. Chen YZ, Guo F, Sun HW, Kong HR, Dai SJ, Huang SH, et al. Association between XPG polymorphisms and stomach cancer susceptibility in a Chinese population. J Cell Mol Med. 2016; 20: 903-8.

40. Ma H, Yu H, Liu Z, Wang LE, Sturgis EM, Wei O. Polymorphisms of XPG/ERCC5 and risk of squamous cell carcinoma of the head and neck. Pharmacogenet Genomics. 2012; 22: 50-7.

41. He J, Wang F, Zhu J, Zhang R, Yang T, Zou Y, et al. Association of potentially functional variants in the XPG gene with neuroblastoma risk in a Chinese population. J Cell Mol Med. 2016. DOI:10.1111/jcmm.12836.

42. Jia WH, Zhang B, Matsuo K, Shin A, Xiang YB, Jee SH, et al. Genome-wide association analyses in East Asians identify new susceptibility loci for colorectal cancer. Nat Genet. 2013; 45: 191-6.

43. Wang F, Zhang SD, Xu HM, Zhu JH, Hua RX, Xue WQ, et al. XPG rs2296147 $\mathrm{T}>\mathrm{C}$ polymorphism predicted clinical outcome in colorectal cancer. Oncotarget. 2016; 7: 11724-32

44. He J, Shi TY, Zhu ML, Wang MY, Li QX, Wei QY. Associations of Lys939Gln and Ala499Val polymorphisms of the XPC gene with cancer susceptibility: a meta-analysis. Int J Cancer. 2013; 133: 1765-75.

45. MacInnes MA, Mudgett JS. Cloning of the functional human excision repair gene ERCC-5: potential gene regulatory features conserved with other human repair genes. Prog Clin Biol Res. 1990; 340A: 265-74.

46. Samec S, Jones TA, Corlet J, Scherly D, Sheer D, Wood RD, et al. The human gene for xeroderma pigmentosum complementation group G (XPG) maps to $13 q 33$ by fluorescence in situ hybridization. Genomics. 1994; 21: 283-5.

47. Friedberg EC, Bond JP, Burns DK, Cheo DL, Greenblatt MS, Meira LB, et al. Defective nucleotide excision repair in xpc mutant mice and its association with cancer predisposition. Mutat Res. 2000; 459: 99-108.

48. Friedberg EC. DNA damage and repair. Nature. 2003; 421: 436-40.

49. O'Donovan A, Davies AA, Moggs JG, West SC, Wood RD. XPG endonuclease makes the 3 ' incision in human DNA nucleotide excision repair. Nature. 1994; 371: 432-5.

50. Wakasugi M, Reardon JT, Sancar A. The non-catalytic function of XPG protein during dual incision in human nucleotide excision repair. J Biol Chem. 1997; 272: 16030-4.

51. Chen Z, Yang J, Wang G, Song B, Li J, Xu Z. Attenuated expression of xeroderma pigmentosum group $C$ is associated with critical events in human bladder cancer carcinogenesis and progression. Cancer Res. 2007; 67: 4578-85.

52. Zhu B, Tian J, Zhong R, Tian Y, Chen W, Qian J, et al. Genetic variants in the SWI/SNF complex and smoking collaborate to modify the risk of pancreatic cancer in a Chinese population. Mol Carcinog. 2015; 54: 761-8.

53. Pardini B, Naccarati A, Novotny J, Smerhovsky Z, Vodickova L, Polakova V, et al. DNA repair genetic polymorphisms and risk of colorectal cancer in the Czech Republic. Mut Res. 2008; 638: 146-53.

54. Mort R, Mo L, McEwan C, Melton DW. Lack of involvement of nucleotide excision repair gene polymorphisms in colorectal cancer. Br J Cancer. 2003; 89: $333-7$.

55. Canbay E, Cakmakoglu B, Zeybek U, Sozen S, Cacina C, Gulluoglu M, et al. Association of APE1 and hOGG1 polymorphisms with colorectal cancer risk in a Turkish population. Curr Med Res Opin. 2011; 27: 1295-302.

56. Liu D, Wu HZ, Zhang YN, Kang H, Sun MJ, Wang EH, et al. DNA repair genes $\mathrm{XPC}, \mathrm{XPG}$ polymorphisms: relation to the risk of colorectal carcinoma and therapeutic outcome with Oxaliplatin-based adjuvant chemotherapy. Mol Carcinog. 2012; 51 Suppl 1: E83-93.

57. Du H, Zhang X, Du M, Guo N, Chen Z, Shu Y, et al. Association study between XPG Asp1104His polymorphism and colorectal cancer risk in a Chinese population. Sci Rep. 2014; 4: 6700. 